\title{
An unconventional dietary ingredient from sea urchin stimulates gonad development in white shrimp (litopenaeus vannamei)
}

\begin{abstract}
Importance of shrimp as a high quality seafood is growing worldwide. Quantities from capture fisheries are unable to meet the human requirement. Aquaculture seeks to bridge the gap between the supply and demand but the aquaculture production is constrained by inadequate availability of high quality seed for farming. While the number of hatcheries producing seed has grown, the problem of egg quality still remains a challenge. The most important factor is broodstock nutrition. Several feeds are availability but failure of gonad maturation and fertility are quite common. This study was carried out to determine the effects of an unconventional diet in the form of sea urchin roe and a commercial feed. The two dietary treatments produced significant $(\mathrm{P}<0.005)$ difference in the gonad development. The gonado-somatic index (\%) of the test specimens offered sea urchin roe was $10.3 \pm 0.5$ while it was $7.0 \pm 1.0$ in the shrimp stock provided the commercial feed. These differences are discussed in this paper.
\end{abstract}

Keywords: Shrimp, Aquaculture, Sea urchin, Unconventional feed, Gonad maturation
Volume 2 Issue 2 - 2015

\author{
Saleem Mustafa, Rossita Shapawi, Saleem \\ Mustafa \\ Borneo Marine Research Institute, University Malaysia Sabah, \\ Malaysia
}

Correspondence: Borneo Marine Research Institute, University Malaysia Sabah, 88400 Kota Kinabalu, Sabah, Malaysia, Tel 60-88-320000,Email saleem@ums.edu.my

Received: November 24, 2014 | Published: April 06, 2015
Abbreviations: GSI, Gonado-Somatic Index; FCR, Food Conversion Ratio; TDL, Total Dietary Lipid; HUFA, Highly Unsaturated Fatty Acids

\section{Introduction}

Shrimp aquaculture is a very important commercial activity which is fairly widespread in developing countries. More than $50 \%$ of the world shrimp production comes from aquaculture and this contributes significantly to global commerce. Asia shares about $80 \%$ of the world's farmed shrimp. Due to increasing demand and stabilization of capture fisheries landings of shrimps, attention has shifted to aquaculture of selected species of this crustacean. However, shrimp aquaculture faces many constraints which have to be addressed. Production of high quality seed in sufficient quantity is absolutely necessary for development of sustainable shrimp farming. It is inconceivable that shrimp culture will expand to meet the seafood market demand without a dependable supply of seed that is healthy and resilient to withstand the challenge posed by pathogens and environmental variability, and is available throughout the year to the farmers.

Shrimp farming relies heavily on wild-caught broodstock conditioned to captivity in the hatcheries and generally subjected to unilateral eyestalk ablation to stimulate maturation for harvesting the eggs. This sort of seed production is susceptible to fluctuations in the availability of gravid specimens in the capture fisheries. Efforts to control the reproductive process for closing the cycle in the hatchery is necessary to reduce the reliance on ready-to-spawn wild stocks from the sea as a source of nauplii. The use of domesticated broodstock in the closed cycle culture as opposed to wild caught broodstock may help in producing pathogen-free larvae. ${ }^{1}$ However, hatcheries often face the problem of gonad maturation and fertility in captive stocks and this becomes a constraint in production of high quality seed.

In the wild, the shrimp selects food from among the diversity of items in the sea and extracts nutrients needed for maturation of its gonads. Whereas in the hatchery, it is offered food that is conveniently available and is economical. Since feeding is generally the most expensive input in land-based aquaculture, efforts are made to formulate diet that is inexpensive to reduce the cost of production and make the product affordable to consumers of seafood. Such a diet may not necessarily comprise the nutrients which can meet the shrimp requirements. It is a common practice to incorporate substantial quantities of ingredients from marine catch in the artificial feed to support growth and maturation in captive shrimp. Currently, aquaculture takes up some $60-80 \%$ of fish meal and $80 \%$ of fish oil produced mainly from the industrial pelagic fisheries and trimmings originating from processing of seafood for human consumption. ${ }^{2}$ This is a big amount and must have significant ecological footprint that makes it an unsustainable practice. While many factors including broodstock condition, genetic composition and endocrinological stimulation determine the egg quality, ${ }^{3}$ broodstock nutrition is a critical factor in sexual maturation, fertility and production of high quality larvae.

Artificially compounded feeds are easier to manage and store in quality needed and pose a lesser risk of pathogenic contamination ${ }^{4}$ but the absence of comprehensive understanding of all that a shrimp needs makes them less effective in sexual maturation compared to natural diets. It is not just the percentages of protein, fat and carbohydrates that matter but a lot more other substances that natural food provides which a compounded ration does not. A class of such substances is that of reproductive hormones. ${ }^{5}$ This is one of the main constraints in aquaculture feed formulation. While efforts are ongoing to fill this knowledge gap for development of a nearly complete feed that provides almost all the ingredients that shrimp needs to develop gonads and provide healthy seed, there is an urgency to lessen pressure on the traditionally exploited prey fish. This study was designed to investigate if diet comprising sea urchin roe can influence the gonad development in white shrimp, Litopenaeus vannamei. The reasons for selecting the sea urchin roe include the unique nature of its chemical composition comprising $\beta$-echinenone and $\beta$-carotene as the major carotenoids and rich amount of glycoprotein that is an 
important component of yolk protein ${ }^{6}$ and substantial amounts of sex steroids, protein, peptidergic factors, and catecholaminergic and cholinergic factors ${ }^{7}$ and many other biochemical constituents whose physiological roles are yet to be investigated. The indication to this effect is the reported influence of lunar cycle on the sea urchin gonad. ${ }^{8}$

\section{Materials and methods}

Two sets of experiments were run in triplicate, each in 1-ton tank with water recirculation system. Sea water was first filtered in DynaSand filter. It is a continuously operating sand filter with an internal washing system that eliminates the need for wash-water storage, tanks, backwater pump and collecting tanks. DynaSand filter operates on the counter-flow principle. The sea water is admitted through the inlet distributor in the lower section of the unit and is cleaned as it flows upwards through the sand bed prior to discharge through the outlet at the upper part of the unit. The water passing through the DynaSand was subjected to biological filtration carried out in a separate tank filled with coral rubble. Average? Throughout the experiment? Water temperature, salinity, dissolved oxygen and $\mathrm{pH}$ was: $26-29^{\circ} \mathrm{C}, 29.8-31 \%, 5.7-6.4$ parts per million and $7.5-7.6$ units respectively. Eighteen specimens of shrimp weighing $65-75 \mathrm{~g}$ body weigh were released in each tank. Shrimp were fed at the rate of $5 \%$ of body weight twice daily in divided shifts: morning $(8 \mathrm{am})$ and evening $(6 \mathrm{pm})$. This was done to avoid wastage of the feed and impairment of the water quality that decomposition of unused feed is known to cause. Feed was placed in the water and shrimp consumed it when it settled at the bottom. Treatment-A comprised the sea urchin roe while treatment-B included feeding a formulated diet (Sanfeng and Sanfapulin feed number 3) which is a commercial pelleted feed containing protein $(42 \%)$, lipid $(16 \%)$, carbohydrate $(27 \%)$ and ash (4\%).Trials lasted 6 weeks after which the shrimp were taken out, weighed and dissected to remove gonads for calculating the gonadosomatic index (GSI) by the formula: GSI = gonad weight $(\mathrm{g}) \times 100 \%$ / body weight (g). During the trials, weight of feed consumed was measured for determining the food conversion ratio.

\section{Results and discussion}

Significant differences $(\mathrm{P}<005)$ were noticed in gonad condition of the shrimp in the two dietary treatments. This was evident from the female gonado-somatic index which averaged $10.3 \pm 0.5 \%$ and in treatment-A fed sea urchin roe and $7.0 \pm 1.0 \%$ in treatment-B offered the commercial pelleted feed. Statistical comparison on between GSIs is missing here. This difference could be due to two reasons: 1) higher digestibility of sea urchin roe and 2) chemical constituents of the roe. Food conversion ratio (FCR) was $1.53 \pm 0.33$ in shrimp fed roe while it was higher $(1.98 \pm 0.03)$ in the stock given pelleted end. Statistical comparison on between FCRs is missing here. This significant difference $(\mathrm{P}<005)$ in the food conversion suggests that the experimental diet higher in digestibility generates more nutrients for assimilation in the body and results in a general improvement in the physiological condition of the body. Certainly, more nutrients are available for gonad development when the shrimp consumed this type of diet. Presumably, the roe also provided hormones and precursors of gonad building material which could have a targeted action on the ovaries of the shrimp receiving a roe ration. While considerable data has been published on the effect of different diets on goad development in white shrimp, the authors are not aware of any study of this nature.

This part is not important for the paper unless the authors provide data on urchin roe composition and references. Other than that it is only speculation. In an earlier investigation ${ }^{9}$ it was demonstrated that the GSI is influenced by total dietary lipid (TDL) levels in the white shrimp. Higher TDL levels led to increase in the concentration of highly unsaturated fatty acids (HUFA). Because HUFA cannot be synthesized de novo by the penaeid shrimp ${ }^{10}$ these should be present in the diet for gonad development and offspring quality. Importance of HUFA in diet of this species of shrimp has also been emphasized by Lytle et al. ${ }^{11}$ who suggested that the profiles of these fatty acids are a comparative tool in assessing the maturation diet. Mendoza et al. ${ }^{12}$ reported the influence of squid extracts on triggering the secondary vitellogenesis in the white shrimp. Ceballos et al. ${ }^{13}$ are of the view that for the balanced nutrition to have effects on somatic growth land gonad development, optimum culture conditions are necessary.

Undoubtedly, this is an interesting area of research that should be thoroughly pursued. Some of the chemicals that could be involved in gonad development include sulphated polysaccharides with unique structures in the roe ${ }^{14}$ and even hormones and pheromones. The nature and identity of these potentially gonad-stimulating substances is still unknown, but most likely include fatty acids, triglycerides, sterols, complex polysaccharides and polypeptides.

The possibility of chemicals similar to pheromones in their function has also been suggested earlier. ${ }^{15}$ The authors have reported dramatic influence of the environmental factors on gametogenesis and nutrient storage and suggested the existence of some sort of hitherto unknown system of communication between environment and reproduction organs. Coordination that seems to exist between gametogenesis and nutrient translocation in the gonad indicates the existence of physiologically controlled and environmentally influenced mechanisms. ${ }^{7}$

\section{Conclusion}

This study suggested that some substitutes for forage fish such as the sea urchin can be effective in supporting gonad development in shrimp. Such ingredients should be identified to lessen pressure on forage fish. The present study will address the serious concerns which are being expressed on the dwindling populations of prey fish in the wild, and one of the major factors is their supply to the aquaculture industry. Marine invertebrates might hold key to discovery of gonadstimulating chemicals. Knowledge of fertility-enhancing biochemical substances is still limited and this offers opportunities for focused investigations on the topic. Success of this trial will probably encourage mass culture of sea urchins for supplying the roe for nourishment of the shrimp broodstock reared in hatcheries for seed production.

\section{Acknowledgments}

None.

\section{Conflicts of interest}

None.

\section{References}

1. Chimsung N. Maturation diets for black tiger shrimp (Penaeusmonodon) broodstock: a review. Songklanakarin J Sci Technol. 2014;36(3):265273 .

2. Obach A. Alternative feed ingredients support continued aquaculture expansion. Global Aquaculture Advocate. 2013;p.26-27.

3. Racotta IS, Palacios E, Ibarra AM. Shrimp larval quality in relation to broodstock condition. Aquaculture. 2003;227:107-130. 
4. Wouters R, Nieto J, Sorgeloos P. Artificial diets for penaeid shrimp. Global Aquaculture Advocate. 2000;3:61-62.

5. Muunpol O, Meejing P, Piyatiratitivorakul S. Maturation diet based on fatty acid content for male Penaeusmonodon (Fabricius) broodstock. Aquaculture Research. 2005;36(12):1216-1225.

6. Unuma T, Suzuki T, Kurokawa T, et al. A protein identical to the yolk protein is stored in the testis in male red sea urchin, Pseudocentrotus depressus. Biol Bull. 1998;194:92-97.

7. Lawrence JM (Ed.), Edible Sea urchins: Biology and Ecology. (2nd edn), Elsevier, BV, Amsterdam, Netherlands. 2007.

8. Juinio-Menez MA, Malay MCD, Bangi HGP. Sea Urchin Grow-out Culture: Coastal Resources Management Tools. Coastal Environment Resources Management Tools. Marine Environment Resources Foundation. The Marine Sciences Institute, University of the Philippines, Diliman, Quezon City, Philippines. 2001.

9. Wouters R, Piguave X, Bastidas L, et al. Ovarian maturation and haemolymphatic vitellogenin concentration of Pacific white shrimp Litopenaeus vannamei (Boone) fed increasing levels of total dietary lipids and HUFA. Aquaculture Research. 2001;32:573-582.
10. Kanazawa A, Teshima SL, Tokiwa S. Nutritional requirements of prawn-VII Effect of dietary lipids on growth. The Japanese Society of Fisheries Science. 1977;43(7):849-856.

11. Lytle JS, Lytle TF, Ogle JT. Polyunsaturated fatty acid profiles as a comparative tool in assessing maturation diets of Penaeus vannamei. Aquaculture. 1990;89(3-4):287-299.

12. Mendoza R, Revol A, Fauvel C, et al. Influence of squid extracts on the triggering of secondary vitellogenesis in Penaeus vannamei. Aquaculture Nutrition. 1997;3(1):55-63.

13. Ceballos-Vazquez, Bertha P, Palacios, et al. Gonadal development in male and female domesticated whiteleg shrimp Litopenaeus vannamei in relation to age, weight and grow-out conditions. Aquaculture. 2010;308(3-4):116-123.

14. Florkin M, Scheer BT. Chemical Zoology. Academic Press, New York and London. Vol 3. 1996.

15. Cinelli LP, Andrade L, Valente AP, et al. Sulfated alpha-L-galactans from the sea urchin ovary: selective 6-desulfation as eggs are spawned. Glycobiology. 2010;20(6):702-709. 